\title{
METODOLOGÍA PARA EL DESARROLLO DE CURSOS VIRTUALES DE APOYO AL APRENDIZAJE COMBINADO EN EL BACHILLERATO UNIFICADO DEL ECUADOR
}

\author{
Methodology for the development of virtual courses to support \\ learning combined in the unified baccalaureate of Ecuador
}

RICARDO PATRICIO MEDINA CHICAIZA[1], WALFREDO GONZÁLEZ HERNÁNDEZ[2]

Recibido: 03 de mayo de 2019. Aceptado: 22 de junio de 2019

DOI: http://dx.doi.org/10.21017/rimci.2019.v6.n12.a63

\begin{abstract}
Resumen
En el artículo se presenta la problemática de diseñar cursos virtuales para el aprendizaje combinado en el bachillerato unificado del Ecuador. Posteriormente se realiza un análisis de las consideraciones acerca de esta problemática desde definir el blearning como forma de organización, el diseño de cursos virtuales y el cómo se diseñan. Posteriormente se desglosan las acciones de la metodología propuesta.

Palabras clave: Diseño de Cursos Virtuales, Aprendizaje Combinado, Metodologías.
\end{abstract}

\begin{abstract}
The article presents the problem of designing virtual courses for combined learning in the unified baccalaureate of Ecuador. Subsequently, an analysis of the considerations about this problem is made from defining the blearning as an organizational form, the design of virtual courses and how they are designed. Subsequently, the actions of the proposed methodology are broken down.
\end{abstract}

Keywords: Journal article, Journal Impact Factor, Research Writing, Research work.

\section{INTRODUCCIÓN}

L AS TICS se han incorporado en el Ecuador como un elemento ajeno a las vivencias de la sociedad, su introducción se produjo, probablemente, como producto de las políticas de comercialización de las transnacionales productoras de bienes tecnológicos. Adicionalmente, el Ecuador se caracteriza por ser un consumidor de la tecnología Informática producida en los países industrializados, tanto a nivel de hardware como de software, siendo el desarrollo de programas educativos con apoyo de software libre aún muy incipiente. La creación de aulas virtuales como proyectos educativos apoyados en tecnología informática y telecomunicaciones, es una actividad multidisciplinaria que demanda la participación de profesionales de la educación y de la telemática. En dicho proceso, es necesario tomar en cuenta diversos elementos, como: la caracterización de la población a la que va dirigida, el diseño del currículo del curso, el desarrollo de materiales educativos, la organización de los

1 Magister en Tecnologías de la Información y Multimedia Educativa. Docente de la Escuela de Ingeniería en Sistemas. Pontificia Universidad Católica del Ecuador. Docente de la Facultad de Ciencias Administrativas. Universidad Técnica de Ambato. Ambato, Ecuador . Correo electrónico: pmedina@pucesa.edu.ec/ricardopmedina@uta.edu.ec

2 Doctor en Ciencias Pedagógicas. Profesor titular de Ingeniería Informática. Universidad de Matanzas, Matanzas, Cuba. Correo electrónico: medina_patricio@yahoo.es 
recursos tecnológicos, el diseño de ambientes de aprendizaje, el entrenamiento a profesores y estudiante, el acondicionamiento de los ambientes físicos, el aspecto financiero y el establecimiento de normas, entre otros.

En el caso específico del bachillerato unificado ecuatoriano, se manifiestan varias insuficiencias en el desarrollo de los cursos en los espacios virtuales de enseñanza aprendizaje como apoyo al aprendizaje combinado, lo cual influye negativamente en el adecuado diseño, estructura, aprendizaje de los estudiantes. En el diagnóstico realizado se revelan las siguientes limitaciones:

1. La primera situación que se detecta mediante la observación directa es que, unos bajos índices de docentes utilizan los entornos virtuales para promover el aprendizaje combinado.

2. No se han detectado metodologías del diseño de cursos virtuales bLearning en los EVEA, lo cual implica desconocimiento en los profesores.

3. Los modelos existentes como ASSURE, ADDIE, Dick and Carey, Morrison Ross y Kemp, son muy generales, y no están orientados a modalidad bLearning en los EVEA y tienen un diseño marcadamente cognitivista.

Por todo lo referido anteriormente, el autor plantea el siguiente problema científico: ¿Cómo desarrollar cursos para el apoyo del aprendizaje combinado en los espacios virtuales de enseñanza aprendizaje de las asignaturas del bachillerato unificado ecuatoriano? Y para responder a este problema científico se trazó el siguiente objetivo: Elaborar una metodología para el desarrollo de cursos en los espacios virtuales de enseñanza aprendizaje como apoyo al aprendizaje combinado de las asignaturas del bachillerato unificado ecuatoriano.

\section{Desarrollo}

El siglo XXI ha contribuido al surgimiento de lo que se denomina la sociedad digital, provocada por el acelerado desarrollo económico, político, social y cultural y dentro de ella, la educación virtual empezó a principios del siglo XXI con el crecimiento acelerado de las empresas punto com (.com), donde se puso de manifiesto la gran cantidad de cursos de pregrado, posgrado, a distancia [1-4]. El bLearning fue luego constituyéndose como una forma de organización en las instituciones de educación presencial, sobre todo en el Ecuador, donde las TIC es su carta de presentación en la oferta académica. Varios especialistas [5-7], asumen el blended learning (BL) como una modalidad en la cual se combina lo positivo de la formación presencial (trabajo directo de actitudes y habilidades) con lo mejor de la formación a distancia (interacción, rapidez, economía). Para la autora Sanaiey [5] esta mezcla de canales de aprendizaje enriquece el método formativo y permite individualizar la formación a cada uno de los destinatarios y cubrir más objetivos del aprendizaje. En esta autora se observa que reduce el bLearning a un método de enseñanza. Para otros autores [8-10], el bLearning es una mezcla de enseñanza o entrenamiento presencial y a distancia, blearning es la educación que incluye presencia física y las interacciones de los estudiantes y los profesores, que utiliza ambientes electrónicos que puedan ser accedidos en cualquier lugar y tiempo. De la misma manera que en las anteriores, no se precisa cuáles son las actividades presenciales y no presenciales.

Como aporte fundamental de sus investigaciones varios autores [11]; [8] señalan que, para combinar los métodos de la enseñanza tradicional y virtual, es necesario crear una nueva metodología que permita adecuar contextualmente esta nueva modalidad, y lograr efectos positivos en el proceso de enseñanza aprendizaje. Después del análisis realizado acerca del aprendizaje combinado, se coincide con los autores referidos en la necesidad de formular definiciones que sean integradoras y que permitan una mejor comprensión del proceso. Así pues, más que una cierta tecnología o método, para esta investigación se asume que el bLearning es una forma de organización del proceso de enseñanza - aprendizaje que presupone el uso intensivo de tecnologías, integrando presencialidad y no presencialidad en dependencia de los objetivos de la organización para la formación de los estudiantes. Esta definición supera las concepciones en las cuales la presencialidad se presupone preponderante sobre 
la no presencialidad al asumirla como una nueva forma de organización que las integra en las cuales se configuran los componentes personales y no personales en una dualidad de espacios.

Otro aspecto relevante en esta definición son los objetivos para la formación de los estudiantes como guía para la integración de las dos formas de organización. Ello presupone la inexistencia de patrones predeterminados en la integración de lo presencial y lo no presencial en el proceso formativo, sino que se configuran a partir de todo el entramado social que se da en la formación de los estudiantes.

\section{Los EsPacios VirTuales de Enseñanza Aprendizaje en EL APRENDIZAJE COMBINADO (BLEARNING)}

En las reflexiones actuales [12]; [9], los EVEA son sistemas informáticos diseñados como espacios virtuales ricos en situaciones que deben animar a los estudiantes hacia el aprendizaje, particularmente los escenarios lúdicos que se puede utilizar en el curso como una estructura de metas en el proceso de aprendizaje. En la última década, los recursos tecnológicos han evolucionado acorde a los equipos informáticos y software para su diseño como: simuladores, evaluadores en línea, video tutoriales, videos interactivos, mapas mentales, ejercicios interactivos en 2D y 3D, documentos dinámicos, foros, chat, multimedia, entre otros [13-16]. Varios autores [17] [18] hacen referencia a que una docencia de calidad es aquella que facilita el aprendizaje de los estudiantes, les ofrece los recursos y los espacios para poder reflexionar de manera autónoma sobre los contenidos de las asignaturas, desarrollar prácticas lo más cerca posible a la realidad con técnicas y tecnologías innovadoras. Sin embargo, otra cuestión necesaria de estudio es el diagnóstico de los estudiantes en un EVEA donde pueden darse niveles mayores de autenticidad. Se reconoce en la literatura consultada en los últimos años [19]; [20] la importancia del diagnóstico y monitoreo de los estilos de aprendizaje de los estudiantes. A partir del diagnóstico es importante destacar el análisis de los tipos de estrategias didácticas propuestos en la literatura [21]; [22]: centradas en la individualización de la enseñanza, para la enseñanza en grupo, centradas en el trabajo colaborativo, centradas en la presentación y la colaboración.

Otro elemento esencial en la no presencialidad de los cursos en la modalidad de aprendizaje combinado es el estudio independiente y su orientación. El estudio independiente, tanto individuales como grupales, relacionados con la planificación del curso en el cual es esencial que el estudiante aprenda a estudiar, reflexione y sistematice los saberes y habilidades adquiridas en el proceso docente - educativo con la utilización de sus métodos y técnicas de estudio de conjunto con la familia y la comunidad [23]. Una de las evidencias de este estudio se encuentra en el portafolio del estudiante pues recoge el resultado de aprendizaje esperado de cada unidad académica, que exista siempre una retroalimentación adecuada, para que el estudiante pueda dar solución a retos planteados de una manera crítica y reflexiva. Para ello pueden utilizarse todas las herramientas de trazabilidad que proveen los EVEAS que permiten controlar cómo ha transcurrido el aprendizaje y la orientación oportuna del profesor en cada momento, sobre todo para el logro de la retroalimentación oportuna cuando se usa la tecnología[24]. Ello no es posible lograrlo sin una adecuada comunicación entre los estudiantes, el grupo y los profesores que imparten las asignaturas, jugando un papel esencial la gestión cooperada de la didáctica [25].

La comunicación sincrónica y asincrónica para la realización de actividades de reflexión, debate o para el apoyo entre estudiantes y profesor es un elemento de vital importancia en el diseño de los cursos en el aprendizaje combinado. Es de especial interés el análisis del curso virtual de aprendizaje ubicuo iconográfico que se diseña bajo una identidad gráfica homogénea y estandarizado basado en una serie de iconografías que sintetizan conceptos o ideas asociadas a una temática específica. Del análisis de esta concepción, se puede corroborar que el uso de imágenes cambia el entorno bajo el cual el estudiante puede generar aprendizaje y lo conduce a un ambiente de descubrimiento y protagonismo [24]. Ello parte de la idea que la representación de los conceptos transcurre a través de imágenes, así como las operaciones principales con ellos se realiza a través de las imágenes de sus representantes, cuestión esencial sobre todo en los EVEA [26] si se quiere el 
desarrollo de los estudiantes en estos espacios y la importancia de los como símbolos que permitan reconocer los procesos que representan [15]; [16]. Muy asociado a la necesidad y uso de las imágenes en los EVEA es la fatiga que se produce por un mal tratamiento de los colores. La ergonomía se encarga de explicar estos fenómenos de fatiga, entre otros, que pueden afectar las interacciones entre los seres humanos y otros sistemas, en el caso que ocupa esta tesis con las tecnologías de la información en un ambiente de aprendizaje [26]. Algunos de los primeros estudios como el realizado por las autoras Pereira Simões and de-Moraesa [27] se centraban en algunas cuestiones como la usabilidad u otras. En estos procesos de interacción juegan un papel fundamental, en especial para el diseño de un EVEA, los criterios de la autora del-Valle Porras [28]. En otros estudios [29] se analizan estos procesos simulando entornos reales donde se causan un alto grado de estrés.

Otra solución comentada con anterioridad es la consideración de un sistema de cursos[30] que surge vinculado al desarrollo de competencias en los EVEA. Para estos autores se sume el Sistema de Cursos Virtuales como “...un conjunto de cursos relacionados entre sí con un hilo conductor sustentado en un EVEA para el desarrollo de cuestiones relacionadas con el aprendizaje, para lo cual con un curso independiente no es posible... responde a las características de aquello que pretende desarrollar y es el objetivo del sistema ... un principio para su desarrollo es la correspondencia entre el SCV y los planes de estudio vigentes para la enseñanza en el nivel que corresponda que determina la organización de los cursos para corresponder con la lógica del saber humano a aprender" [31].

El sistema de cursos, al decir de estos autores, puede combinarse con el aprendizaje basado en proyectos que permite la evaluación integral del aprendizaje [30]. El bachillerato, a diferencia del entorno universitario para el cual fue creado el $\mathrm{SCV}$, no ofrece a los estudiantes una formación profesional, sino que sienta las bases para esta desde la formación de una cultura general integral. Por tanto, es parte de la concepción de los cursos en un EVEA la determinación de cuáles se integrarán en sistemas y cuáles quedarán de manera independiente.

\section{IMPLEMENTACIÓN DE CURSOS EN LOS EVEA PARA SUSTENTAR EL APRENDIZAJE COMBINADO}

Al implementar un curso bLearning, no se trata de diseñarlo y ponerlo en un computador; se debe realizar una correcta combinación de recursos, actividades, comunicación, interactividad y brindar un apoyo permanente a las actividades presenciales [32]. Para otro autor Mateos [33] la gestión de los recursos educativos pasa por las siguientes fases: la creación, validación, publicación, catalogación - organización-publicación, la búsqueda - localización - recuperación, utilización, reutilización; cada una de estas aristas involucra a procesos, herramientas y personas con roles diferentes. Esta concepción es más abarcadora que las precedentes pues encierra tanto la producción de recursos como el capital humano involucrado en él, sin embargo, no se aprecia como parte de esta gestión la comunicación entre los departamentos involucrados.

En relación con el diseño del curso se distinguen varios elementos didácticos necesarios para tener en cuenta, que para varios autores [34]; [35] deben ser: objetivos claros y expectativas para todos, gestión efectiva de aulas virtuales, respeto mutuo, rigor académico, discusión interactiva de alta calidad para fomentar el pensamiento crítico, fomentar la participación auténtica de los estudiantes y estilos de enseñanza flexibles y diferenciación para diversos estilos de aprendizaje. Sin embargo, no se aprecia en estos autores otros elementos didácticos que deben ser tomados en cuenta como el diseño de la evaluación, la importancia de las estructuras funcionales del curso, los materiales que deben ser colocados, así como los métodos de enseñanza que deben ser considerados para el aprendizaje combinado soportado por las tecnologías. Otra de las cuestiones esenciales desde una perspectiva didáctica en un curso de aprendizaje combinado es, sin duda, la evaluación. Para Colomé Cedeño [36], los diferentes tipos de evaluación pueden confluir en un EVEA y contribuyen al cumplimiento de las funciones de la evaluación. Para el logro de estos propósitos evaluativos en un EVEA es necesario la retroalimentación oportuna, individualizada y eficaz de los errores cometidos por los actores involucrados en el proceso siempre ajustado al nivel de desarrollo conseguido por el estudiante. Al mismo tiempo, los EVEAS pueden suponer una oportunidad

Rev. Ingeniería, Matemáticas y Ciencias de la Información Vol. 6 / Núm. 12 / julio - diciembre de 2019; pág. 13-24 
para mejorar la visibilidad del proceso de aprendizaje del alumno, lograr un mayor seguimiento de este, y mejorar el ajuste de la ayuda pedagógica que ofrece el profesor, haciéndola más personalizada, diversificada y adaptada a las necesidades de cada situación. Por tanto, la clave se sitúa en los usos didácticos que se realice de los recursos tecnológicos por los participantes. De esta manera, la atención en el diseño de los cursos de aprendizaje combinado debe situarse en el uso efectivo de la tecnología que hacen los participantes para permitir a miembros más expertos el profesor o compañeros más expertos en la planificación de las influencias educativas para el despliegue de las sucesivas tareas de evaluación. De esta manera, la tecnología será una herramienta útil en la medida que permita al profesor y a los estudiantes utilizarla en las prácticas de evaluación.

En concordancia con Chero [37] los factores institucionales tienen como referente principal para dicha adaptación a los factores pedagógicodidácticos se basan en tener coherencia entre el modelo de enseñanza - aprendizaje, los factores tecnológicos direccionados hacia la usabilidad, interfaz intuitiva y amigable y los factores personales que se asientan entre los proyectos individuales o grupales según sea aplicada a la actividad que se aplique. También es preciso para la implementación de los cursos virtuales el análisis del capital humano y técnico que constituyen la base fundamental para dicho proceso, los cuales deben estar aptos para el desarrollo de los cursos en los cuales estén asignados.

Por tal motivo, en la implementación de los cursos virtuales juegan su papel los procesos administrativos refiriéndose a los objetos corporativos, la estrategia de desarrollo para obtener la una máxima utilidad, los costos mínimos para la implantación que permitirá delinear estrategias y los objetivos que son la base fundamental para una correcta introducción de un entorno virtual hacia la comunidad educativa según sea el contexto [38]. Debido a lo antes expuesto, y considerando la opinión de varios autores consultados [39] se asume que la implementación adquiere más relevancia en la práctica de la propia actividad docente, debido a que el material didáctico para ser estudiado es transmitido por el docente y ampliado, en algunos casos, por el entorno virtual, y cuyos materiales son evaluados por los logros de los estudiantes.
Asumiendo los aspectos tratados hasta el momento se asume la implementación de cursos virtuales para el aprendizaje combinado como como una fase de su desarrollo, orientado a un conjunto de operaciones y rutinas dirigidas a concluir esta etapa que son realizados por un sistema de actores para el cumplimiento de los objetivos del programa de estudios, con los objetivos de la institución y sus contenidos organizados en sistema de asignaturas o de una asignatura en particular presentando diversas funcionalidades para un proceso BLearning. Este proceso de desarrollo se caracteriza por el enfoque de sistema que se establece entre sus componentes principales: las relaciones entre los sujetos participantes, la organización de la formación integral del estudiante, los componentes del proceso en el EVEA y las actividades presenciales, enfocado en las herramientas y metodologías que se adoptan para garantizar la producción y una adecuada utilización durante la enseñanza de las asignaturas, así como los procesos de evaluación de cada una de las fases.

\section{CONFECCIÓN Y ESTRUCTURACIÓN DE LA METODOLOGÍA POR ETAPAS}

\section{Objetivo general}

Contribuir al desarrollo de cursos para el apoyo del aprendizaje combinado en los espacios virtuales de enseñanza aprendizaje de las asignaturas del bachillerato general unificado ecuatoriano.

\section{Fundamentación}

La metodología se construye sobre la base de los fundamentos de las Ciencias de la Educación como la Filosofía, la Sociología, la Psicología, la Pedagogía y la Informática, los cuales permiten tanto teórica como metodológicamente su organización científica. En la realización de tareas docentes desde los entornos virtuales, los estudiantes interactúan con los conocimientos de la asignatura y los elementos tecnológicos, a la vez que transforman sus conocimientos. En el orden psicológico, la propuesta se sustenta en el Enfoque Histórico Cultural del desarrollo humano [40].

En lo pedagógico la metodología facilita el desarrollo de un proceso de formación inicial dinámico, flexible y desarrollador, centrado en el 
desarrollo integral de la personalidad y de los entornos virtualizados, en el que se manifiestan las categorías sobre las instrucciones y la educación como una unidad dialéctica, con un marcado enfoque profesional pedagógico; en el que se asumen los principios, leyes, categorías y funciones de la Pedagogía y la Didáctica General en estrecha relación con las didácticas particulares de cada ciencia. La metodología para implementación del aprendizaje combinado en los espacios virtuales de enseñanza aprendizaje de las asignaturas del bachillerato general unificado ecuatoriano que se propone posee las siguientes características: Carácter multidimensional, procesual y dinámico; Carácter flexible; Carácter sistémico; Interdisciplinar y transdisciplinar. Para el logro del objetivo propuesto se proponen un sistema de etapas que constituyen el aparato instrumental de esta metodología y para ello se sistematizan las acciones contenidas en varias metodologías de tesis que la han antecedido[31].

\section{A. Etapa Primera. - Diagnóstico de la situación actual para la implementación del aprendizaje combinado.}

En esta etapa se analizan las acciones para diagnosticar el estado actual para la implementación de cursos en el EVEA para sustentar el aprendizaje combinado. En este momento se determinan las necesidades de superación de los implicados para implementar y habilitar de forma independiente las herramientas complementarias y dar mantenimiento la plataforma, lo que servirá de base para la modelación de las acciones que se acometerán durante la etapa de implementación.

\section{Objetivo de esta etapa}

Diagnosticar las condiciones iníciales para la implementación la implementación de cursos en el EVEA para sustentar el aprendizaje combinado.

Para el desarrollo de esta etapa se han previsto las siguientes acciones:

Elaboración de instrumentos para conocer el grado de conocimiento en la utilización y creación de los cursos virtuales de aprendizaje que poseen los actores del proceso de enseñanzaaprendizaje en el bachillerato; Aplicación de instrumentos que permitan obtener información sobre el grado de desarrollo de las habilidades del trabajo con las TIC en el proceso de enseñanza - aprendizaje y de la preparación de los profesores de cada una de las asignaturas y de los estudiantes para la interacción con las TIC; Realización de un curso inicial para diagnosticar el empleo de las TIC, en especial los entornos virtuales de aprendizaje y el aprendizaje combinado; Diagnóstico de la infraestructura: se debe diagnosticar las condiciones tecnológicas para la implementación de un entorno virtual de aprendizaje, los equipos con servidores, la red, la existencia de sistemas informáticos; Diagnóstico sobre el aprendizaje combinado: Se diagnostica el conocimiento acerca del aprendizaje combinado y sus características que les permita una correcta conducción de este proceso. Ello es importante pues permitirá conocer el estado inicial de la preparación de los profesores para enfrentar el diseño de cursos para el aprendizaje combinado.

Se sugiere desarrollar un curso inicial con los profesores con el objetivo de sensibilizarlos con este tipo de trabajo. Para ello es necesario precisar su alto significado desde el punto de vista educativo, considerando que se trata de involucrar un número de asignaturas que son complementarias para lograr el objetivo principal del presente escrito.

\section{B. Etapa Segunda: Preparación de las condiciones iniciales para la implementación de los cursos virtuales que sustente el aprendizaje combinado.}

\section{Objetivo de esta etapa}

Organizar las condiciones iniciales para dar solución a las dificultades encontradas en la primera etapa que permita implementar convenientemente los cursos en un EVEA que sustente el aprendizaje combinado.

En esta etapa se contribuye a la implementación cada uno de los entornos de virtuales a ser creados, teniendo en cuenta la búsqueda de las tecnologías educativas y herramientas adecuadas de manera que sensibilicen y prepararen a los involucrados sobre el cambio educativo que se requiere y lo que implica para los cursos en un EVEA que sustente el aprendizaje combinado. Para el desarrollo de esta etapa se han previsto las siguientes acciones: Análisis de los resultados del diagnóstico realizado para estructurar una pro-

Rev. Ingeniería, Matemáticas y Ciencias de la Información Vol. 6 / Núm. 12 / julio - diciembre de 2019; pág. 13-24 
puesta de solución para la superación de los profesores; Desarrollo de un proceso de socialización e intercambio de información entre los profesores de las diferentes carreras y asignaturas que se imparten en el año; Propiciar un alto nivel de comunicación y de relaciones entre los involucrados, elemento este que facilitará el estudio de los temas; Profundización en las relaciones interdisciplinarias que puedan ser determinadas y la integración de diferentes componentes a través de discusión en grupos de trabajo; Implementación de un sistema de superación de los profesores utilizando variadas vías; Revisión de la documentación asociada a ejecuciones anteriores de la metodología para socializar los errores cometidos, utilizarlos como recursos de aprendizaje y las soluciones que se han encontrado como vía para facilitar el trabajo de los equipos de desarrollo de cada curso.

La ejecución de las acciones anteriormente explicitadas posibilitará que las estructuras de dirección seleccionen las variantes de superación para cada profesor. El colectivo de profesores analiza, organiza y desarrolla el trabajo práctico en función de la metodología que se propone, de forma que exprese los cambios para el desarrollo de los cursos virtuales y el entorno de aprendizaje.

\section{Etapa Tercera: Implementación de los cursos virtuales que sustente el aprendizaje combina- do en el bachillerato unificado.}

\section{Objetivo de esta etapa}

Diseñar los contenidos para la implementación de los cursos que sustente el aprendizaje combinado para el bachillerato unificado.

En esta etapa los docentes especialistas en cada área deben desarrollar los contenidos a ser implementados en las aulas virtuales. La conformación de los equipos, no en todos los casos, será una variante para garantizar que cada colección que se implemente en los cursos virtuales le sea asignado un encargado y ellos implementarán las acciones de diseño de los contenidos. Los usuarios (rol profesor) tendrán acceso para modificar solo a las colecciones que fueron desarrolladas por ellos. Para el desarrollo de esta etapa se han previsto las siguientes acciones:
Realización de convocatorias para la participación de los profesores; Preparación de los contenidos para los cursos; Determinación del objetivo de cada usuario relacionado con la unidad temática en la cual va a trabajar; Selección del estándar para el desarrollo del curso virtual para garantizar la interoperabilidad en las plataformas.

Esta etapa, según Fernández López [41], es importante porque en ella se deben de reconocer los objetivos institucionales para determinar qué tecnología debe poseer el entorno virtual. Es en este momento que se diseña y se crea el equipo de trabajo que va a construir, implementar, enfocar, desplegar y evaluar los cursos virtuales para el aprendizaje combinado. Para la elaboración de cada recurso de aprendizaje que sea implementado por los profesores es importante tener en cuenta estas etapas definidas por Hernández Alfonso [42] $\mathrm{y}$ adecuadas a los requerimientos de esta investigación: 1. Fase Preparatoria; 2. Formulación del tema y verificación de su relevancia; 3 . Conformación del equipo de producción; 4 . Formulación tentativa de los objetivos; 5 . Investigación temática y pre alimentación; 6 . Elaboración de la estructura y determinación de las especificidades; 7. Fase de elaboración.

En el caso de seleccionarse un sistema de cursos virtuales entonces es importante realizar otros análisis. Para ello es necesario retomar las acciones propuestas por los autores [30] realizando las adecuaciones pertinentes al objetivo de esta metodología.

El objetivo de esta etapa sería diferente: Determinar una metodología para el diseño del SCV que sustente el aprendizaje combinado en el bachillerato ecuatoriano. Para el desarrollo de esta etapa se han previsto las siguientes acciones: Desarrollar aspectos visuales para incrementar su presencia del espacio virtual de aprendizaje; Planificar el alcance de un aula virtual, decidir la practicidad del aula virtual teniendo en cuenta su integración con lo presencial y otros cursos. Definir estándares y marcas académicas. Concretar habilidades y destrezas a desarrollar. Categorizar las aulas virtuales propias; Determinar el Alcance Académico; Determinar el Alcance Experimental y el Alcance Tutorial. Para ello se debe entender claramente que el tiempo que se tarde en planificar curso virtual, decidir la practicidad del curso virtual, definir 
estándares y marcas académicas, concretar habilidades y destrezas a desarrollar y categorizar el uso de las aulas virtuales propias.

Es importante destacar que en el caso que sea seleccionado el SCV para el sustento del aprendizaje combinado, pues es necesario establecer los procesos de integración de éstos. El objetivo de la subetapa sería: Integrar los cursos desarrollados en un SCV para el sustento del aprendizaje combinado en el bachillerato.

Para el desarrollo de esta etapa se han previsto las siguientes acciones: Desarrollo de las interfaces de gráficas por temas y asignaturas; La integración realizada apoyará a los profesores en un plan de enseñanza en la medida en que permita y facilite la gestión de contenidos y la selección de materiales educativos digitales adecuados, garantizará el acceso a materiales educacionales confiables y permitirá la reactualización del sistema de aulas virtuales; El usuario (rol profesor) va a gestionar en el sistema de archivos solamente un curso para lo cual será el administrador de su curso; El usuario Coordinador del curso va a gestionar el sistema de cursos. Según ACM/IEEE [43], las normas de estandarización para un correcto desarrollo de software se basan en el modelo o metodología de desarrollo de software, en el SCV creado se tiene como precedente que influirá en el correcto uso de estos estándares. Es necesario, además, en el caso del sistema de cursos la Verificación y Validación del SCV.

El Objetivo de esta subetapa es: Establecer las acciones de verificación y validación del SCV como recurso de aprendizaje.

Esta etapa está contemplada dentro de la metodología con vistas a realizar la validación y verificación del SCV. Para ello se organiza un equipo de probadores compuesto por los profesores de mayor experiencia, el jefe de departamento y el coordinador de carrera. Para el desarrollo de esta etapa se han previsto las siguientes acciones: Diseño de un plan de pruebas y auditoría que permita estructurar cada una de las pruebas, los resultados esperados y los casos de prueba que se utilizarán; Ejecución del plan diseñado para detectar los errores cometidos; Depuración de los errores detectados; Documentación del proceso de depuración.
D. Etapa Cuarta: Aseguramiento de la calidad de los cursos virtuales que sustente el aprendizaje combinado en el bachillerato unificado.

En esta acción de la metodología vuelve a plantearse la elección de un SCV o cursos virtuales de manera separada. Se analizará en primer lugar el objetivo y las acciones necesarias en el primer caso y posteriormente el objetivo y las acciones necesarias en el segundo caso.

\section{Objetivo de esta etapa}

Establecer las acciones de aseguramiento de la calidad del SCV como recurso de aprendizaje para sustentar el aprendizaje combinado en el bachillerato unificado.

De tal modo los procesos relacionados a los estudiantes están interconectados directamente con los procesos relacionados con la gestión y la gestión del aprendizaje, en donde se enmarcan los procesos de desarrollo de software que el egresado ya los visualizo en toda su vida estudiantil. Acciones a realizar: Revisiones periódicas del cumplimiento de los parámetros de calidad definidos en cada curso y en el SCV en la cual participan los directivos educativos y los administradores de la plataforma; Establecimiento de acciones para análisis sobre el cumplimiento de los estándares de calidad propuestos; Realización de encuestas para medir el nivel de satisfacción de los estudiantes con cada curso en particular por parte del profesor y el sistema de cursos por parte del directivo al cual se subordinan las asignaturas que serán integradas; Selección de los criterios a tener en cuenta para los cambios necesarios. En el caso de ser seleccionado un curso por asignatura entonces el objetivo sería: Establecer las acciones de aseguramiento de la calidad cada asignatura como recurso de aprendizaje para sustentar el aprendizaje combinado en el bachillerato unificado.

E. Etapa Quinta: Aseguramiento del soporte a los cursos virtuales implementados que sustente el aprendizaje combinado en el bachillerato unificado

\section{Objetivo de esta etapa}

Establecer las acciones de aseguramiento a la implementación de los cursos para el sustento del aprendizaje combinado.

Rev. Ingeniería, Matemáticas y Ciencias de la Información Vol. 6 / Núm. 12 / julio - diciembre de 2019; pág. 13-24 
Una vez que los cursos se encuentran en el EVEA y se utilizan como parte de las actividades es necesario cumplimentar acciones que permitan su uso por los actores del proceso docente educativo. Este proceso se define en los procesos de desarrollo de software como soporte de los sistemas informáticos instalados en la organización [44] y juega un papel importante pues aseguran la continuidad de su uso por los profesores y estudiantes. De ahí el por qué estos procesos de soporte pueden utilizar algunas de las etapas anteriores como la primera y la segunda de esta metodología.

Acciones a realizar: Revisiones periódicas de actualizaciones en los EVEAs que impliquen nuevas funcionalidades que se reviertan en mejoras para el aprendizaje combinado; Actualización de los profesores y resto de personal involucrado en la gestión de los cursos que les permita utilizar los recursos disponibles de forma eficiente y eficaz para así mejorar los procesos de aprendizaje combinado; Análisis de la necesidad de mantenimiento o retiro de los cursos que se encuentran en el EVEA instalado sobre la base de las solicitudes de los directivos autorizados al efecto; Realización de encuestas para medir el nivel de satisfacción de los estudiantes y profesores con cada con las actualizaciones propuestas en el EVEA.

F. Etapa Sexta: Mantenimiento o reciclaje de cursos virtuales implementados que sustente el aprendizaje combinado en el bachillerato unificado

\section{Objetivo de esta etapa}

Establecer las acciones de mantenimiento a la implementación de los cursos para el sustento del aprendizaje combinado.

Otras de las acciones a realizar una vez instalado el EVEA e implementado los cursos que sustentan el aprendizaje combinado es la modificación de éstos o su cambio por otros que sean necesarios. Este proceso se define en los procesos de desarrollo de software como mantenimiento de los sistemas informáticos instalados en la organización [45] y son importantes pues aseguran las modificaciones a los cursos utilizados por los profesores y estudiantes.

Acciones para realizar: Revisiones periódicas de las necesidades de actualización o cambio en los cursos implementados en el EVEA; Diseño de las modificaciones previstas; Implementación de las acciones de modificación que sean necesarias teniendo en cuenta el alcance de cada una de ellas; Realización de encuestas para medir el nivel de satisfacción de los estudiantes y profesores con cada con las modificaciones propuestas para cada curso.

\section{G. Etapa Séptima: Retiro de los cursos virtuales que sustente el aprendizaje combinado en el ba- chillerato unificado.}

\section{Objetivo de esta etapa}

Establecer las acciones de retiro de los cursos para el sustento del aprendizaje combinado.

Las transformaciones educativas pueden llevar a modificaciones curriculares que impliquen, a su vez, eliminación o integración de cursos como procesos de rediseño curricular [46] que tienen lugar en el bachillerato. Este proceso se define en los procesos de desarrollo de software como retiro de los sistemas informáticos instalados en la organización [45] y son importantes pues aseguran que se acceda a cursos acordes con las causas que originaron los procesos de rediseño curricular. Una vez detectado que existen cursos que deben ser eliminados o integrados en el EVEA pues el directivo a cargo de la conducción de los procesos de rediseño curricular debe dirigir un oficio a los gestores del EVEA.

Acciones para realizar: Análisis de los procesos de rediseño curricular y su impacto en los cursos implementados en el EVEA; Proponer sistemas alternativos; Instalar los sistemas alternativos.

\section{H. Etapa Octava: Evaluación de las acciones an-} teriores de la metodología para la implementación de los cursos virtuales que sustente el aprendizaje combinado.

\section{Objetivo de esta etapa}

Establecer las acciones de evaluación, seguimiento y control de todas las etapas que se integran en la metodológica elaborada para valorar los resultados formativos y el desempeño profesional pedagógico e incorporar las medidas necesarias la implementación del SCV como recurso de aprendizaje. 
Esta etapa está contemplada dentro de la metodología con vistas a realizar la evaluación de la efectividad de las etapas anteriores. Para ello se procederá a una nueva aplicación de las técnicas utilizadas en la constatación inicial y se analizarán los datos para medir el impacto de la metodología. En todas las acciones previstas participan los equipos de desarrollo y el investigador, en cada una de ellas se levanta un acta de los aspectos esenciales tratados. Para el desarrollo de esta etapa se han previsto las siguientes acciones: Realización de encuentros periódicos con los equipos de profesores seleccionados para conocer los criterios acerca del proceso y obtener valoraciones que permitan perfeccionarlo; Valoración y autovaloración de los logros y dificultades en cuanto a conocimientos y formas de implementación de las acciones concebidas para la implementación del sistema de cursos virtuales con todos los involucrados; Valoración del desarrollo alcanzado en la implementación del sistema de cursos virtuales, esto se realizará al validar utilizando para ello el examen de acreditación profesional implantado por la Secretaria Nacional de Ciencia y Tecnología de Ecuador; Determinación de la calidad del cumplimiento de la metodología elaborada, así como de su efectividad a partir del desempeño de los profesores, así como de la calidad del entorno virtual.

La metodología propuesta se diferencia cualitativamente de otras por la integración de varias fases que no se encuentran en otras metodologías por el propio origen de ella. Esta metodología se concibe para la implementación de un sistema de cursos virtuales y sus correspondientes cursos creados por profesores de una institución educativa en función del desarrollo del alumno. Derivado de esta concepción de la metodología se articulan en sus etapas y procedimientos la superación de profesores, la especialización de estos y su integración en equipos de trabajo y la posterior evaluación del trabajo realizado.

\section{CONCLUSIONES}

El análisis de la literatura especializada permitió establecer el marco teórico que sustenta el diseño de cursos en los EVEA han sido objeto de estudio de varias corrientes y tendencias, desde diferentes perspectivas científicas. De modo general, se considera al diseño de un curso en los EVEA particularmente en la semipresencialidad como una unidad funcional y estructural compleja que atraviesa diferentes fases de desarrollo. De ahí la importancia de la acción consciente del profesor para lograr su mejor desempeño. Con base al análisis de diferentes autores sobre el término bLearning, se propone una nueva definición enfocandose al aspecto de institucionalidad. Se pone de manifiesto la importancia del tratamiento de la estructura en el diseño de un curso en los EVEA para el aprendizaje combinado. Además, al elemento social y estético. La dinámica del diseño de un curso en los EVEA, asume particularidades que responden a las nuevas formas de gestión, tecnológicas, pedagógicas, estructural, social y estéticas que caracterizan a estos entornos, se tiene en cuenta estas características para proponer una definición de diseño de cursos en los EVEA.

La metodología que se propone parte de la concepción expresada en los fundamentos teóricos y tiene el propósito de contribuir la implementación de curso de apoyo al aprendizaje combinado para la formación de la competencia DSW. La metodología está organizada a partir de dos núcleos esenciales: el primero, de carácter teórico, donde se definen los fundamentos que lo sustentan; el segundo, de carácter metodológico, que está referido a su concepción estructural - funcional para la implementación del curso. Otro aspecto esencial es la integración de los profesores en equipos de desarrollo que interactúan a través de proyectos multidisciplinarios.

\section{REFERENCIAS}

[1] V.Heckler, Experimentation in Sciences in Distance Education: online inquiry with teachers in the Virtual Learning Environment (VLE). (Doutor em Educação em Ciências pela Universidade Federal do Rio Grande), Universidade Federal do Rio Grande, Rio Grande. 2014.

[2] C. C. Pataca, V. E. Sentí \& S. R. P. Medina, Retos para la introducción de la enseñanza a distancia en el ISUTIC. Revista EDUCATECONCIENCIA., 5(6), 135- 145. 2015.

[3] L. A. Tomei, Lexicon of Online and Distance Learning. New York, USA: Rowman \& Littlefield Education. 2010.

[4] G. Veletsianos, Emerging Technologies in Distance Education (G. Veletsianos Ed.). Edmonton, Canadá: AU Press, Athabasca University. 2010.

Rev. Ingeniería, Matemáticas y Ciencias de la Información Vol. 6 / Núm. 12 / julio - diciembre de 2019; pág. 13-24 
[5] N. Z. Sanaiey, The comparative study of the effectiveness of using e-learning, blended learning and presence learning in continuous medical education. WORLD JOURNAL OF MEDICAL SCIENCES, 10(4), 488-493. 2014.

[6] A. L. Whiteside, Introducing the social presence model to explore online and blended learning experiences. Journal of asynchronous learning network. 2015.

[7] T. Yigit, A. Koyun, A. S. Yuksel \& I. A. Cankaya, Evaluation of Blended Learning Approach in Computer Engineering Education. Procedia - Social and Behavioral Sciences, 141, 807 - 812. 2014.

[8] L. García Aretio, Blended learning y la convergencia entre la educación presencial y a distancia. RIED. Revista Iberoamericana de Educación a Distancia, 21(1), 09-22. doi:10.5944/ried.21.1.19683. 2018.

[9] Á. Torres-Toukoumidis, L. M. Romero-Rodríguez \& A. Pérez-Rodríguez, Ludificación y sus posibilidades en el entorno de blended learning: revisión documental. RIED. Revista Iberoamericana de Educación a Distancia, 21(1), 95-111. doi:10.5944/ ried.21.1.18792. 2018.

[10] S. Vanslambrouck, C. Zhu, B. Pynoo, V. Thomas, K. Lombaerts \& J. Tondeur, An in-depth analysis of adult students in blended environments: Do they regulate their learning in an 'old school' way? Computers \& Education, 128, 75-87. doi:10.1016/ j.compedu.2018.09.008. 2019.

[11] H. Al-Samarraie \& N. Saeed, A systematic review of cloud computing tools for collaborative learning: Opportunities and challenges to the blendedlearning environment. Computers \& Education, 124, 77-91. doi:10.1016/j.compedu.2018.05.016. 2018.

[12] L. I. R. Rojas \& F. J. García-Peñalvo, Methodological proposal for massive training of Ecuador's civil servants, in the educational field, through MOOC courses on virtual learning environments. 705-713. doi:10.1145/3012430.3012596. 2016.

[13] J. Cabero-Almenara, C. Llorente-Cejudo \& J. J. Gutiérrez-Castillo, Evaluación por y desde los usuarios: objetos de aprendizaje con Realidad aumentada. Revista de Educación a Distancia (RED)(53). doi:10.6018/red/53/4. 2017.

[14] J. L. Jurado \& H. M. Bustamante, Método de especificación de patrones colaborativos para plataformas de ciencia, un enfoque desde la gestión de conocimiento. Campus Virtuales, 6(1), 23 - 37. 2017.

[15] F. B. Topu \& Y. Goktas, The effects of guidedunguided learning in $3 \mathrm{~d}$ virtual environment on students' engagement and achievement. Computers in Human Behavior, 92, 1-10. doi:10.1016/ j.chb.2018.10.022. 2019.
[16] L. Yu-Ju, I. Y. T. Hsiao \& S. Mei-Feng, Effective Learning Design of Game-Based 3D Virtual Language Learning Environments for Special Education Students. Educational Technology \& Society, 21(3), 213-227. 2018.

[17] E. Berrocal de Luna \& S. Megías Ruiz, Indicadores de calidad para la evaluación de plataformas virtuales. TEXTOS. Revista Internacional de Aprendizaje y Cibersociedad, 19, 234- 245. 2016.

[18] R. Marciniak \& J. G. Sallán, Dimensiones de evaluación de calidad de educación virtual: revisión de modelos referentes. RIED. Revista Iberoamericana de Educación a Distancia, 21(1), Preprint. doi:10.5944/ried.20.2.16182. 2017.

[19] P. Nascimento, R. Barreto, T, Primo, T. Gusmão, T., \& E. Oliveira, Recomendação de Objetos de Aprendizagem baseados em Modelos de Estilos de Aprendizagem: Uma Revisão Sistemática da Literatura Paper presented at the VI Congresso Brasileiro de Informática na Educação (CBIE 2017), Brasil. 2017.

[20] R. A. C. Ortuño, E. M. M. Morgado \& C. M. O. Rodríguez, Diseño de Objetos de Aprendizaje adaptados para cuatro estilos de aprender: un estudio de caso. Educação e seus sentidos no mundo digital, 25(2), 548-572. 2016.

[21] R. S. Contreras Espinosa, J. L. Eguia Gómez \& L. Solano Albajes, Investigación-acción como metodología para el diseño de un serious game. RIED. Revista Iberoamericana de Educación a Distancia, 19(2), 71-90. doi:10.5944/ried.19.2.15624. 2016.

[22] H. O. Stynze Gómez, Diseño de estrategia de aprendizaje colaborativo basado en el uso de dispositivos móviles. Revista Evaluación Educativa, 5(1), 1 14. 2016.

[23] Y. Rodríguez Pérez, Estrategia pedagógica para la dirección del estudio individual en la educación secundaria básica. (Doctor en Ciencias Pedagógicas), Universidad de Ciencias Pedagógicas "Juan Marinello Vidaurreta", Matanzas. 2012.

[24] R. P. Medina-Chicaiza, M. C. Cruz-Escobar \& W. González-Hernández, Espacio virtual iconográfico de aprendizaje ubicuo orientado al desarrollo del pensamiento lógico en bachillerato general unificado. Revista Electrónica Formación y Calidad Educativa (REFCalE), 5(2), 85 - 98. 2017.

[25] R. R. Ramírez Oyarzo, Estrategia metodológica para el desarrollo de la competencia comunicativa profesional en idioma inglés en la licenciatura en periodismo- Universidad Tecnológica Equinoccial del Ecuador. (Tesis presentada en opción al Grado Científico de Doctor en Ciencias Pedagógicas.), Matanzas. 2013. 
[26] S. Coomans \& G. S. Lacerda, PETESE, a Pedagogical Ergonomic Tool for Educational Software Evaluation. Procedia Manufacturing, 3, 5881-5888. doi: 10.1016/j.promfg.2015.07.895. 2015.

[27] A. Pereira Simões \& A. de-Moraesa, The ergonomic evaluation of a virtual learning environment usability. Work, 41, 1140-1144. doi:10.3233/WOR2012-0293-1140. 2012.

[28] Y. del-Valle Porras, Recomendaciones para el diseño de ambientes virtuales de aprendizaje a partir de la evaluación de aspectos ergonómicos. (Trabajo de ascenso para optar a la categoría de Profesor Asistente), Universidad Nacional Experimental Politécnica "Antonio José de Sucre", Caracas-República Bolivariana de Venezuela. 2017.

[29] T. Nowicki, J. Koszela \& ?. Matuszela?ski, Virtual reality environment to study the ergonomic features of firefighters' activity. Paper presented at the MATEC Web of Conferences. 2018.

[30] L. A. Llerena Ocaña, Sistema de cursos virtuales para la formación de la competencia desarrollar sistemas web en la Carrera de Sistemas de la Universidad Regional Autónoma de los Andes UNIANDES. (Doctor en Ciencias de la Educación), Universidad de la Habana, Ciudad de la Habana, Cuba. 2017.

[31] L. A. Llerena Ocaña \& W. González Hernández, Nuevo sistema de cursos virtuales como aporte al desarrollo de las competencia profesionales para desarrollar sistemas web. Avanzada Científica, 20(2), 1 - 17. 2017.

[32] E. B. Durán, M. Álvarez, S. Unzaga, N. Salazar, B. Fernández Reuter, G. González, R. D. Cordero, Diseño y desarrollo de aplicaciones de aprendizaje ubicuo. Paper presented at the XVIII Workshop de Investigadores en Ciencias de la Computación (WICC 2016), Entre Ríos, Argentina. 2016.

[33] J. E. G. Mateos, Estrategia de gestión de recursos educativos abiertos en forma de objetos de aprendizaje en la universidad de la habana. (Doctor en Ciencias de la Educación), Universidad de La Habana, Ciudad de la Habana. 2010.

[34] R. T. Cuenca \& L. G. Á. Hernández, Análisis del diseño de cursos virtuales con reader y tablet en la universidad de holguín. Tecnología Educativa, Vol. 2 / Núm. 1 / enero-junio de 2017 |(1), 62 - 71. 2017.

[35] S. Pertuz \& J. Torres, Lineamientos para el diseño de Cursos Online Masivos y Abiertos (MOOC) en Ingeniería Electrónica1. Entre Ciencia e Ingeniería, 11(21), 42-49. 2017.

[36] D. M. Colomé Cedeño, Ambiente de Trabajo para la Producción de Objetos de Aprendizaje en la Educación Superior. (Tesis presentada en opción al grado científico de Doctor en Ciencias Técnicas), Universidad de las Ciencias Informáticas, Ciudad de la Habana, Cuba. 2014.

[37] M. J. S. Chero, Implementación de un sistema de gestión del aprendizaje para los colegios secundarios de Utcubamba-Amazonas. TZHOECOEN, 9(1), 70-80. 2017.

[38] A. Ortega-Arranz, L. Sanz-Martínez, S. ÁlvarezÁlvarez, J. A. Muñoz-Cristóbal, M. L Bote-Lorenzo, A. Martínez-Monés \& Y. Dimitriadis, From LowScale to Collaborative, Gamified and Massive-Scale Courses: Redesigning a MOOC. Paper presented at the European Conference on Massive Open Online Courses. 2017.

[39] L. Umek, D. Ker •i?, A. Aristovnik \& N. Toma • evi?, An assessment of the effectiveness of Moodle elearning system for undergraduate public administration education. International Journal of Innovation and Learning, 21(2), 165-177. 2017.

[40] L. S. Vygotsky, Pensamiento y lenguaje. Teoría del desarrollo cultural de las funciones psíquicas. Argentina: Ediciones Fausto. 1995.

[41] J. A. Fernández López, M. M. Pintor Chávez \& Gómez M. G. Zermeño, Development of policies for a digital repository for a higher education institution with technological disadvantages. education policy analysis archives, 24, 21. 2016.

[42] R. Hernández Alfonso, Material Didáctico Digital para contribuir al aprendizaje de la asignatura Análisis de Datos en los estudiantes de segundo año del curso por encuentros de Licenciatura en Cultura Física. (Máster en Educación SuperiorMención: Docencia Universitaria), Universidad de Matanzas, Matanzas. 2017.

[43] ACM/IEEE, C., Society (Producer). Association for Computing Machinery. Association for Computing Machinery. Retrieved from https://www.acm. org/. 2017.

[44] R. Martin Monroy, R. Ribon \& P. Puello, A Methodological Approach for Software Architecture Recovery. Indian Journal of Science and Technology, 11(21), 1-8. doi:10.17485/ijst/2018/ v11i21/124487. 2018.

[45] D. E. Casaro, P. L. Alfonzo, S. I. Mariño \& M. V. Godoy, Mantenimiento Correctivo Aplicado a un Sitio Basado en Joomla. Una Propuesta Centrada en la Accesibilidad. Revista Latinoamericana de Ingeniería de Software, 3(2), 101-107. 2015.

[46] O. F. Mero Jalca, M. V. Tapia Zúñiga \& M. P. Ramos Rodríguez, Rediseño curricular de la Carrera de Ingeniería Forestal en la Universidad Estatal del Sur de Manabí, Ecuador. Revista Conrado, 13(59), 212-221. 2018.

Rev. Ingeniería, Matemáticas y Ciencias de la Información Vol. 6 / Núm. 12 / julio - diciembre de 2019; pág. 13-24 